\title{
Reproduce the Architectural Landscape Based on Computer Virtual Real- ity Technology
}

\author{
Jinyu Wang ${ }^{1, *}$ and Nirlep Thakkar ${ }^{2}$ \\ ${ }^{1}$ Hohhot Vocational College, Hohhot 010051, China \\ ${ }^{2}$ L.P.S. Institute of Cardiology, G.S.V.M. Medical College, Kanpur, India
}

\begin{abstract}
This paper starts from the concept of computer virtual reality technology, mainly discusses the three main features and the complex technical elements of the virtual reality technology, introduces the key technology of virtual reality technology in architectural landscapes, and analysis of the benefits of virtual reality technology in architectural landscape applications. The so-called computer virtual reality technology (Virtual Reality Technology, referred to VR technology) is used to make simulation and conduct research on the reality things through the computer network technology. As one of the supports of digital city, the computer virtual reality technology has extremely important significance to reproduce the architectural landscape. It is safe, low cost, and almost free from the constraints of environmental conditions technology, which can change time or the proportion of dimensions at any time according to the willingness. The technology has become the common tool for the integration of the design, operation, and analysis and evaluation system in the current architectural landscape design. This paper takes the Zhujiang New Town virtual system designed by Guangzhou Urban Planning Survey and Design Institute as the example, which focused on the specific implementation of the architectural landscape of virtual reality technology, with the objective of evaluating the virtual reality system of that project, namely the application advantage and disadvantage of virtual reality technology.
\end{abstract}

Keywords: Architectural landscape, Research and application, Virtual environment, Virtual reality technology.

\section{INTRODUCTION}

Virtual reality technology originated in the late twentieth century in America, is a highly integrated information technology. It is mainly simulated by a computer to calculate the real construction site environment, performing the virtual environments in specific ranges through a realistic Trinitarian feel of audio-visual and touch. Users can experience a real-time traveling process in a simply completely do not exist environment, which is a system generated proximity immersive experience.

Virtual reality technology is being used since the 1980s in the United States, during which the digital model of the relevant architectural landscape was launched and was the focus of the study. The main field of application is the application of virtual reality technology in architecture, landscape and other projects [1]. Currently, it began to explore landscape architecture design by the virtual reality technology. For example, since the beginning of the establishment of the environment and facilities with hardware and software for virtual reality systems including appropriate building exterior, building structure, landscape, landscape little scene, research architectural landscape works in a virtual environment roaming form three viewpoints. In the field of landscape architecture in our country, the current application and research on virtual reality technology is still ongoing.

*Address correspondence to this author at Hohhot Vocational College, Hohhot, 010051, China; Tel: +8613848918903;

E-mail: 823454045@qq.com in its nascent stage of development. The application of virtual reality technology research projects in the country is still rarely precarious been involved. In short, the foundation theory research and application of virtual reality technology in the landscape architecture field needed to be further improved [2].

Due to the immaturity of virtual reality technology, the application in architectural landscapes for now is still very limited. Especially in our country, at present, mainly the technical means used were three-dimensional modeling and visual simulation and other, which can show the overall program visually in the computer. The biggest benefit is that it can help the relevant designers take the post-assessment and audit of the programs more intuitively for variety of planning and designing programs. At present, there is more advanced technology abroad that has been able to achieve a virtual roaming in the virtual reality system, which can be a more intuitive experience planning result.

Therefore, this paper intends to discuss the relevant theoretical results of virtual reality technologies: First, virtual reality technology and its features, followed by the key technologies in the construction landscape, and then discusses the advantages and significance of virtual reality technology used in the construction of the landscape. This paper takes the building construction of new city Zhujiang Guangzhou as an example of virtual reality, explore the implementation steps of virtual reality technology in architectural landscape, to ensure that the general public has a deeper level of understanding on virtual reality technology and its application in 
the architectural landscape. Thus, this paper discusses the contribution of the virtual reality technology more widely and effectively used in the architectural landscape.

\section{VIRTUAL REALITY TECHNOLOGY}

\subsection{Virtual Reality Technology and Its Features}

Virtual reality technology is used the computer to generate a simulated virtual reality and lifelike interactive dynamic interface, where one can take cross interactive information exchanges on a virtual entity of landscape architecture virtual objects through visual, auditory, tactile, and olfactory senses, to produce an immersive lifelike experience. It also has an inquiry, analysis, evaluation, decision-making, and many other executable features. Virtual reality technology has three basic characteristics: cross-interaction, realism and the degree of association.

Cross-interaction refers to the operate degree of particular geographical environment and the natural degree that the user can feedback in virtual environment, with the effectiveness feature. This cross-interaction is mainly achieved by special equipments such as helmet display, data gloves, etc.

Realism, also known as immersive sense, refers to the degree coefficient of the perception for the real situation of users in the virtual environment, mainly to make users feel that they are part of the virtual environment, and can be immersed in the virtual environment to experience.

Association level refers to the thinking behaviors such as the logic reasoning or associate through subjective sense of designers, a variety of information acquired in virtual environment, the dynamic behavior of their design staff in a virtual system, the ability to imagine the initiative related to its own development with the change of operating status of system.

\subsection{Applications of Virtual Reality in the Architectural Landscape}

\subsubsection{The Combination of Virtual Reality Technology with the Architectural Landscape}

With virtual reality technology continues to mature and the deepening of application in architecture landscape, the requirements of the continuity and forward-looking of architecture landscape in recent years have continued to improve. Landscape architecture field has been the most pressing need of the reality virtual technology. Users can also make the evaluation and assessment by rationality of the perception of space through the various planning programs designed to build a virtual environment, which can avoid the cost and time consumed in the actual implementation of the program, which has important role to improve the efficiency of architectural landscape. The biggest benefit of the application of virtual reality technology in the landscape architecture field is to make up the form of performance weaknesses for traditional architecture and landscape design. First, the user, including the design and planning staff can take the interactive experience in a virtual three-dimensional geographical environment activity in order to facilitate assessment and program evaluation or modification plan, thus greatly improving the program approval rate. Second, it can display the contents of the design programs for assessment or the public. Taking scientific, objective and reasonable interpretation of the development change process in landscape architecture, will help reproduce architectural landscape design plan through virtual reality technology, which can reduce or avoid the more unreasonable planning and design solutions that brought huge losses [3].

\subsection{Related Applications of Virtual Reality Technology}

\subsubsection{Three-Dimensional Modeling Techniques}

In order to achieve a web-based three-dimensional virtual world, we must establish a large number of the corresponding three-dimensional model in architectural landscape design. The establishment of 3D model is the infrastructure project of the entire virtual reality world.

$3 \mathrm{D}$ modeling technology in virtual reality technology is mainly divided into two categories: geometric digital modeling and behavioral modeling. Geometric modeling is the simulation performance based on digital object geometry and shape characteristics, which takes the question graphical data as the main research. Behavioral modeling suggests the comprehensive description on track movement and behavior of objects, which is often referred to animation. More commonly used modeling tools for virtual reality systems include 3D Max, Maya, and reator [4].

Users can roam online and achieve multi-user interaction using the 3D virtual space based on architectural landscape design, and can also give advice to the construction development planning of shown results and the post-modification measures.

\subsubsection{Human-computer Interaction Technology}

In the virtual space of the computer system, the virtual reality technology is the cross interaction technology integrated with the human ear, mouth, eyes, gestures and other senses. It is a highly integrated emerging information technology, which focuses on the integration of digital image processing technology, multimedia technology, sensor technology, computer graphics and other aspects of information technology. Its research goal is to eliminate barriers between people and the environment of the computer. That is the human-computer interaction technology under virtual environment.

\subsubsection{Real-time Dynamic Rendering Technology}

Virtual reality systems were required to generate corresponding graphic images with the trajectory change of user activity. That is, the most important feature of virtual reality technology system is that people can feel the dynamic characteristics of the scene changes at random cross interactions Shown in Fig. (1). Currently, three-dimensional graphics generation technology is quite mature, so the key question is, 


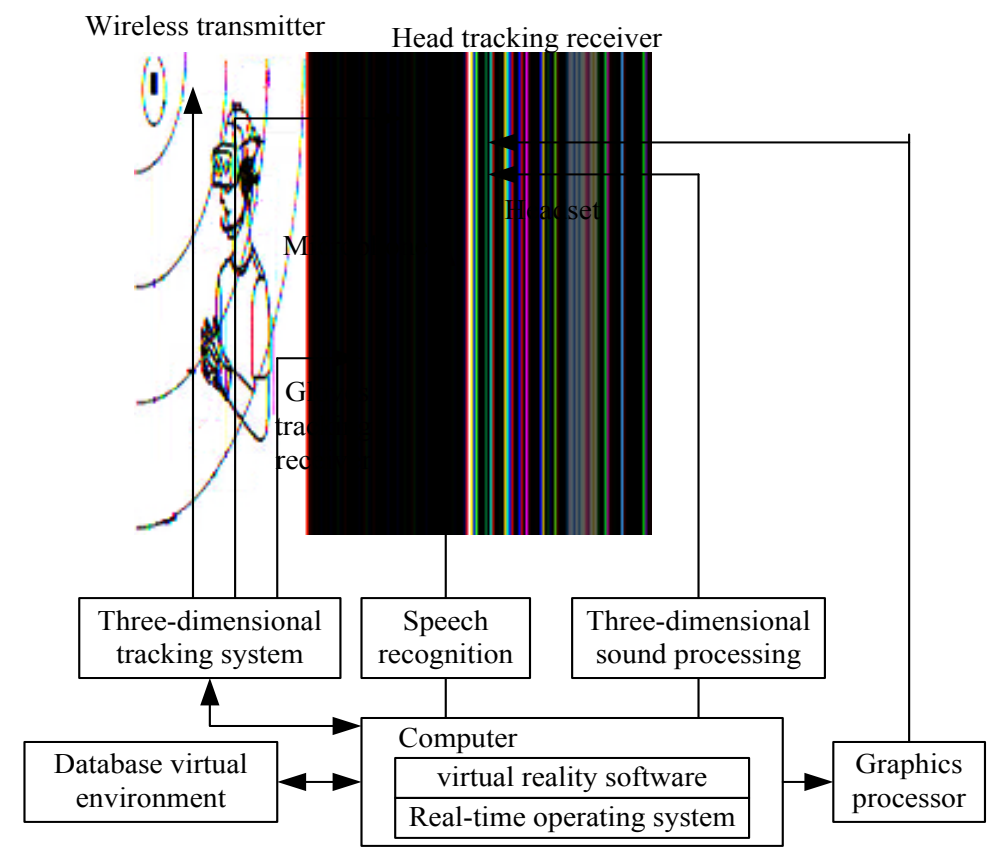

Fig. (1). Sensor technology and three-dimensional display technology.

how to achieve real-time technology model. The virtual environment is the most typical big scene in the architectural landscape, the process of data processing is difficult to achieve real-time rendering due to the large amount of data. So it often requires high-performance configured computers to implement, such as the computer graphics workstations.

\subsubsection{Sensors and Three-Dimensional Display Technology}

Development of virtual reality technology is affected by many factors, mainly based on the common progress of sensor technology and stereoscopic display technology. The main methods to achieve three-dimensional display are roughly four ways on the current market: stereo method, the integration of imaging, voxel method and display technology based on holographic. In essence, the first three methods are not the strict sense three-dimensional information technologies, and the holographic display technique is based on the original way of reproducing the object prototype, the detailed three-dimensional storing information. The digital holographic three-dimensional display technology provided the ideal visual medium for public during the multimedia presentations i.e. the scientific rapid virtual prototyping technology [5].

\section{EMPIRICAL CASE: NEW CITY IN ZHUJIANG GUANGZHOU}

Under these theories, this paper introduces the main implementation steps of virtual reality technology in architectural landscape of the new city building simulation systems in Zhujiang, which is designed by Guangzhou city construction survey and design institute.

Virtual simulation system of Zhujiang new city used virtual reality technology and three-dimensional visualization technology merger to establish the three-dimensional virtual simulation system. Its establishment of virtual simulation system is based on two-dimensional data, planning outcomes data and other data, the entire system can achieve crossinteractive program operation. First, establish the unique 3D scenes through the Creator Terrain Studio of United States leading companies MultiGen-Paradigm in the field of threedimensional simulation, material production tools modeling software, which mainly played the role in establishing threedimensional model of the Zhujiang new city. Then, Vega software environment was used for take the model-driven and functional development and further analysis of the VC programming languages. Overall structure of the simulation platform of Zhujiang new city is shown as Fig. (2).

\subsection{Regional Overview of Example}

The location of Zhujiang new city is really special; it is located in Tianhe district Guangzhou, which is one of China's three major cities, located in the intersection of Haizhu, Tianhe and Yuexiu. The east is the south china express line, west is Guangzhou Avenue, near by the Pearl River, north is the Huangpu Avenue. The Zhujiang new city began to develop in 1992 and land size was 6.2 square kilometers. Superiority of urban space is an important reason for the dramatical change. Due to the reform and opening up of the Zhujiang new city, the power of the rapid economic development of Guangzhou led the formation of a new business center, which gradually formed a unified business atmosphere taking Tianhe sports center as the center region, which began to build a new city development path, not only throughout the southern building skyscrapers group, but also connected to the west traditional business area - Yuexiu district and eastern regions to be developed. The location can be said to be unique and impeccable. The Western area of Zhujiang new city in planning is mainly the business office; the eastern area is mainly the casual living residence. There 


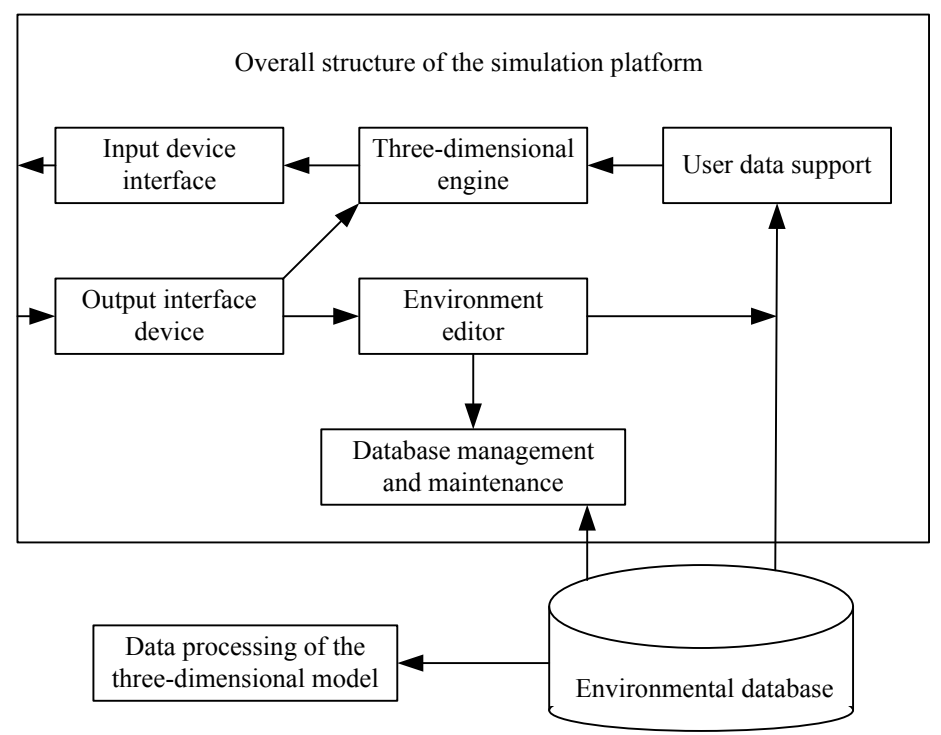

Fig. (2). Overall structure of the simulation platform of Zhujiang new city.

are many proposed projects at the intersection of the city's new central axis of Guangzhou and Zhujiang landscape, such as the Guangzhou opera house, Guangzhou tower and other buildings [6].

\subsection{Facilities Selection of the Software and Hardware of Virtual Simulation System}

Hardware environment: using high-configuration PC, the purpose is to ensure a good operation. Human interface device used a large screen monitor, keyboard, and mouse of the $\mathrm{PC}$ as the basic configuration.

The configuration of machine running:

Pentium IV;

Memory $512 \mathrm{M}$;

HDD 128 GB;

Clocked at $2.4 \mathrm{G}$;

Graphics card GeForce 4600.

Software environment: two major operating systems: Windows NT operating system, Microsoft access database system. tor;

Three-dimensional modeling software: MultiGen Crea-

Software programming tools: Visual $\mathrm{C}++6.0$.

\subsection{Acquisition Conversion Work of Data}

Data collection refers to the process that scanned and digitized into photogrammetric software of the building aerial photographs of Zhujiang new city and through the edge look, image segmentation techniques and other methods to regenerate the image filtering. Then, solid ground model is used to get orthophoto map and keeping this, measured attribute data of the architecture model on the Zhujiang new city basis. Finally, the converting analysis for a variety of obtained data was done to transfer it into the virtual modeling software with different categories.

\subsection{Virtual Scene Modeling and Post Rendering}

Currently, we can directly use Open GL to establish the appropriate modeling tools for specialized development and grouped models using modeling library. But one of the disadvantages of this, is a lot of work. Another method is to use Auto CAD, 3D Max and other common modeling tools to build three-dimensional models, and ultimately by programming or with other aids to transfer the model into the simulation vista [7].

During the buildings, structures and other virtual scene modeling process, the advised software are CAD, 3D max, GIS software, the virtual reality modeling language and so on, which are used for the production of three-dimensional entities. In addition to the three-dimensional entity, it also contains three-dimensional information of objects, such as the light direction, light source characteristics, materials texture, gloss, transparency, and the scene information.

\subsection{Evaluation and Thinking of Virtual Simulation Sys- tem in Zhujiang New City}

Designers and planners can use the above methods and implementation steps provided in this article to build the virtual tour system of Zhujiang new city in a sense. The hardware and software requirements which in a complex virtual reality technology, are relatively high. We could eventually build the main function of these systems in practice, which focused on the visual display and query processing, but was unable to reach the true sense of neglect of roaming. As shown in Fig. (3), the show function is the performance of the zoom rotation and marching animated features in arbitrary. It also can travel along on any path and view the current model by using a mouse, which can also be a two-dimensional space map [8]. 


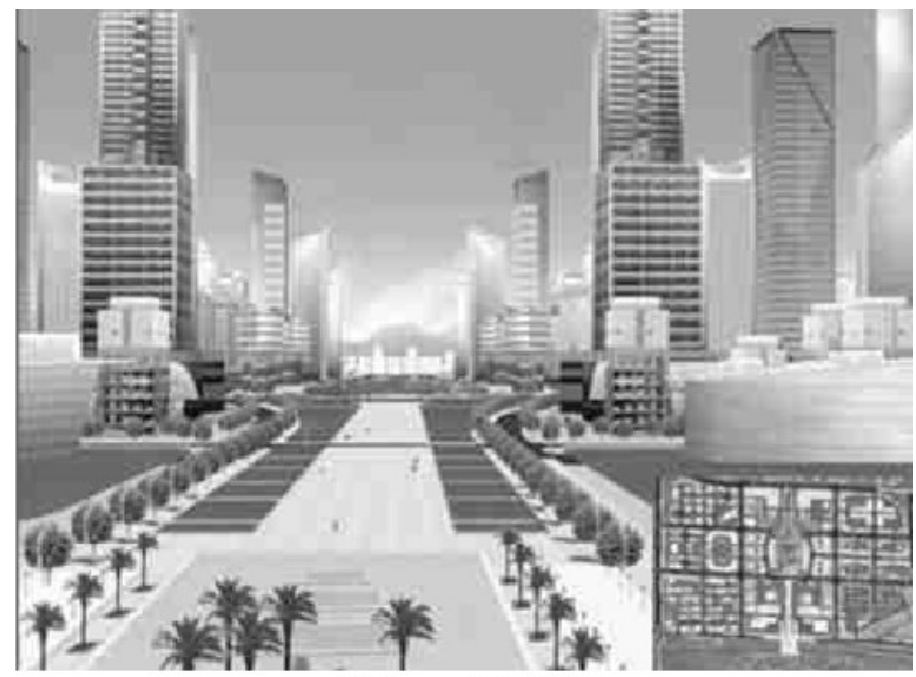

Fig. (3). Dimensional navigation.

Query data in the property and checking out the selected target information based on the database provided information, is one of the main functions of the system; the query includes the name of the construction, the construction area, construction units and the completion time and other information.

As to the showing effect, the system not only can make a true record of the landform features, spatial location and the three-dimensional outer contour of the key sections of every building in Guangzhou Zhujiang new city, but also can directly reflect the overall appearance features of Zhujiang new city, which allowing the public to the visual effects. Thus, Zhujiang new city can be a complete evaluation program. Another advantage of the program is the visual comparison of simulate program [9].

However, this paper argues that virtual reality systems often need to deal with three-dimensional scene of architectural landscape with large-area and large size, which involves collection, storage, analysis, search and replace, display updates and other aspects of treatment for a lot of data, which also means that the establishment and management of data information during the process of building a virtual reality system has become the primary decision-making problem to be solved. In order to solve this problem, people should do further exploration and study of virtual reality technology.

However, the establishment of virtual city system requires a lot of manpower, material and financial resources, which does not only require to show simulate real for a specific time or geographic, but also the management services for follow-up design and construction. This requires taking into account the establishment of virtual reality system, and must take into account the compatibility issues in existing planning data information systems and information management systems, which can help project managers make planning approval and management decisions more accurate, fair and efficient [10].

Finally, virtual reality technology, computer graphics, and virtual reality systems, all of them have even greater hardware requirements. Planners must find hardware platforms for virtual reality systems through discussion and practice.

\section{CONCLUSION}

With the in-depth and extensive application of virtual reality technology in landscape architecture, virtual reality technology has played a positive role in promoting sound for the application of architectural landscapes, improvement at the scientific level of landscape architecture and promoting the development and construction of digital cities and other aspects. With the development of computer technology, virtual reality technology and network technology, virtual reality technology provides greater efficacy for the scientific nature of landscape architecture and design.

However, there are many issues still not resolved under the current level of development; such as the virtual reality technology in the field of landscape architecture in our country warrant further in-depth applications. The main reasons can be summarized as follows: technical aspects in general, cover a wide range of relevant geographic planning, which makes it hard to imagine a three-dimensional model data demand, which makes the relevant three-dimensional modeling and data processing workload is too large. As regards, hardware and software requirements, for 3D modeling software the current market limitation was large, which is just good at establishing a three-dimensional model of the building, while a large amount of three-dimensional landscape architectural model suitable for its own software and brand is not sufficient, especially it is relatively narrow on the domestic market. In addition, the hardware and software requirements for the implementation of virtual reality technology are very high. In a sense, a truly complete virtual reality systems often require a long period of work, sometimes a few months even years, which brings huge human and material capital investment cost. As per the standardization, it is imperative to set up a system related with virtual reality technology for data processing and management information as soon as possible. Due to the lack of a unified evaluation criteria forthe information management related to technical 
data, standardization has become the major requirement for virtualization construction of digital virtual development current, which is seen important to create a virtual reality system in different situations at any time to achieve sharing, exchanging, and updating.

\section{CONFLICT OF INTEREST}

The authors confirm that this article content has no conflict of interest.

\section{ACKNOWLEDGEMENTS}

Declared none.

\section{REFERENCES}

[1] Y. D. Liu, Digital Architecture, Dalian University of Technology Press: Dalian, 2002.

[2] M. Liang, Z. P. Liu, and P. R. Zhang, "Implementation of virtual reality system in the dam system", Computer Applications, no. 8, pp. 43-45, 1999.
[3] W. Chen, W. Feng, and Z. Y. Jiao, "Virtual reality technology and riverside landscape study", Building Journal, no. 6, pp. 9-12, 2001.

[4] J. L. Shen, "Applications of virtual reality technology in engineering design", Scientific and Technological Exchanges, no. 3, 2004.

[5] H. H. Xie, Construction Engineering System Simulation, Science Press, Beijing, 2001.

[6] X. J. Hu, Basement and Application of Virtual Reality Technology. Beijing University of Posts and Telecommunications Press, Beijing, 2009.

[7] X. X. Li, Virtual Reality Technology and Art, Wuhan University Press, Wuhan, 2007.

[8] Z. L. Liu and B. G. Zhang, "Application study of virtual reality technology in urban planning", Beijing Surveying and Mapping, no. 4, pp. 1-3, 2008

[9] F. S. Wu and Z. G. Pan, "Application of virtual reality technology in urban planning and design", China Institute of Metrology, vol. 13, no. 4, pp. 9-14, 2002.

[10] X. H. Zhu and B. Xiao, "Virtual reality technology and its applications of geographic sciences", Nanjing Normal University, vol. 22, no. 3, pp. 105-109, 1999.

Received: September 16, 2014

(C) Wang and Thakkar; Licensee Bentham Open.

This is an open access article licensed under the terms of the Creative Commons Attribution Non-Commercial License (http://creativecommons.org/licenses/by-nc/4.0/) which permits unrestricted, non-commercial use, distribution and reproduction in any medium, provided the work is properly cited. 